\title{
PgCYP76B93 docks on phenylurea herbicides and its expression enhances chlorotoluron tolerance in Arabidopsis
}

\author{
Jinhoon Jang ${ }^{1 \dagger}$, Sanjida Khanom ${ }^{1 \dagger}$, Youngkook Moon ${ }^{2 \dagger}$, Sooim Shin ${ }^{2}$ and Ok Ran Lee ${ }^{1^{*}}$ (C)
}

\begin{abstract}
The phenylurea herbicides are used to control annual and perennial weeds on crop cultivating fields. The excessive usage of these agrochemicals increase many environmental problems. Thus, engineering transgenic plant for herbicide metabolism can provide efficient and eco-friendly means for enhanced phytoremediation capacity. Cytochrome P450 enzymes comprise one of the major plant enzyme families that mediate the oxidative degradation of xenobiotic chemicals, including herbicides. Considering these notions, phytoremediation properties of transgenic ginsengderived PgCYP76B93 in Arabidopsis to phenylurea herbicides were assessed. Phylogenetic tree of PgCYP76B93 clustered in between close to the herbicide metabolism-related enzyme families and terpenoid biosynthesis-related. The expression of PgCYP76B93 was considerably upregulated upon treatment with phenylurea herbicide, chlorotoluron. Simulated docking using Autodoc program predicted possible interaction with chlorotoluron. Transgenic Arabidopsis plants overexpressing PgCYP76B93 were resulted in slightly reduced plant height with relatively small leaves. The lower plant height in the PgCYP76B93-overexpressing line than in the control revealed that it was linked to the expression of gibberellin oxidases (GAox). The bioassay of transgenic plants growing on herbicide-containing media revealed enhanced resistance against chlorotoluron.
\end{abstract}

Keywords: Panax ginseng, Cytochrome P450, Herbicide, Chlorotoluron, Phytoremediation

\section{Introduction}

Plant cytochrome P450 enzymes (CYPs) are involved in catalyzing various types of monooxygenation/hydroxylation reactions in primary and secondary metabolism [1]. One third of all known CYP enzymes are from the plant kingdom, and the number of enzymes is estimated to be up to $1 \%$ of the total number of annotated plant species. This implies that diversification within CYPs has led to the emergence of new metabolic pathways throughout land plant evolution.

\footnotetext{
${ }^{*}$ Correspondence: mpizlee@jnu.ac.kr

†Jinhoon Jang, Sanjida Khanom and Youngkook Moon contributed equally to this work

${ }^{1}$ Department of Applied Plant Science, College of Agriculture and Life

Science, Chonnam National University, Gwangju 61186, Korea

Full list of author information is available at the end of the article
}

A newly diversified species-specific secondary metabolic pathway in Panax ginseng Meyer involves ginseng saponins, also called ginsenosides [2, 3]. Considering the pharmaceutical efficacies of ginsenosides, studies on ginseng plants largely focused on ginsenoside biosynthesis $[3,4]$. Among the 116 reported P450 genes [5], two CYP genes have been reported to be involved in the dammarane-type of ginsenoside biosynthesis $[6,7]$ and one $C Y P$, called $\beta$-amyrin 28-oxidase, is involved in the oleananetype of ginsenoside biosynthesis [8]. However, besides their physiological roles in secondary metabolism, other important features of CYPs are their detoxification roles against herbicide [9]. The mechanism of acquiring increased herbicide metabolism has not yet been adequately demonstrated. A perennial plant, such as ginseng, has been grown commercially for up to 6 years or even more, and it can be exposed to herbicides over 
several years. Continuous exposure to herbicides might be involved in the diversification of more CYP family genes. Thus, taking the concept one step further, the functional characterization of other $C Y P$ family members from ginseng might shed light on herbicide metabolism.

Modern herbicides contributed a lot to sustainable global food production by minimizing time to remove weeds and substituting destructive soil cultivation [10]. Thus understanding herbicides resistance mechanism is a major challenge for modern agriculture [10]. In higher plants, the herbicide detoxification genes have accumulated through the evolution of multigene families, like glutathione S-transferase [11] or glycosyl transferase [12] and cytochrome P450 enzyme families [9]. The CYPs are by far the largest family of enzymes that mediate the oxidative degradation of xenobiotic chemicals, including herbicides. The CYPs are heme protein that gains electrons from NADPH to catalyze the formation of a molecule of water and an oxygenated product. Xenobiotics such as aromatics, pesticides, and hydrocarbons are usually synthesized for industrial and agricultural purposes. Since some of them are harmful to living organisms, organisms have evolved efficient system such as CYPs to eliminate xenobiotic absorption. Genetically engineered plants for herbicide metabolism provide efficient and eco-friendly means for enhancement of detoxification of harmful substances [13].

The first evidence of the involvement of plant P450 in the metabolism of the herbicide monuron was reported in the microsomal fraction of cotton seedlings by Frear in 1969 [14]. To date, several CYP family members have been characterized as being involved in herbicide metabolism. CYP76B1 was isolated from Jerusalem artichoke (Helianthus tuberosus) and was found to metabolize herbicides belonging to the phenylurea class [15-17]. CYP71A10 was isolated from soybean and determined to metabolize the phenylurea herbicide, chlortoluron, when expressed in yeast, although with an efficiency 10 times lower than that of CYP76B1 [18]. The CYP76C1, $C Y P 76 C 2$, and $C Y P 76 C 4$ genes also provided suitable evidence for herbicide resistance in Arabidopsis [17]. When human-derived CYP1A2 was overexpressed in Arabidopsis, it also increased tolerance to the phenylurea herbicide linuron [19]. Very recently ginseng-derived CYP736A12 was reported to be involved in chlortoluron and isoproturon tolerance when overexpressed in Arabidopsis [20].

In this study, we report another ginseng-derived $C Y P$ gene annotated as $P g C Y P 76 B 93$ that are involved in herbicide tolerance. Homology based molecular docking of PgCYP76B9 shows the more possible interaction with chlortoluron rather than isoproturon. Transgenic plants overexpressing PgCYP76B93 displayed relatively reduced plant height and herbicide chlortoluron tolerance with respect to control lines. The observed functions of $P g C Y P 76 B 93$ provide insight into its use as a selectable marker for genetic engineering of multifunctional crops having the ability to detoxify agrochemicals and environmental contaminants.

\section{Materials and methods \\ Plant materials and growth conditions}

Panax ginseng Meyer 'Chun-Poong' was used as the plant material in this study. Arabidopsis thaliana (genotype Col-0) was used as a heterologous system. Mature Arabidopsis seeds were surface-sterilized for $5 \mathrm{~min}$ in $70 \%(\mathrm{v} / \mathrm{v})$ ethanol and then rinsed three times with sterile water. The Arabidopsis seeds were sown on 1/2 MS medium (Duchefa Biochemie, Haarlem, Netherlands) containing $0.5 \mathrm{~g} / \mathrm{L}$ MES (2-[ $N$-morpholino] ethanesulfonic acid, $1 \%$ sucrose, and $0.8 \%$ phytoagar); the $\mathrm{pH}$ was adjusted to 5.7 using $\mathrm{KOH}$. The seed-sown Petri plates were cold-treated for 2 days and then incubated under long-day photoperiod conditions $-16 \mathrm{~h}$ light and $8 \mathrm{~h}$ dark-at $23^{\circ} \mathrm{C}$ in a growth chamber.

\section{Sequence analysis}

The ginseng EST clones were analyzed by using the BLAST (Basic Local Alignment Search Tool) program against a specialized cytochrome P450 database (https:// drnelson.utmem.edu/CytochromeP450.html). The amino acid sequences were analyzed using the online programs (https://www.ncbi.nlm.nih.gov/Structure/cdd/wrpsb.cgi) and the ProtParam tool (https://web.expasy.org/protp $\operatorname{aram} /$ ). The amino acid sequences were aligned using the BioEdit program (version 7.1.9). A phylogenetic tree was generated according to the neighbor-joining method of the MEGA6 (version 6.06) program.

\section{Chemical treatments in ginseng}

To record gene expression levels with the treatment of herbicides, $10 \mu \mathrm{M}$ of chlorotoluron (CAS Registry No. 15545-48-9, Cayman, USA) and isoproturon (CAS Registry No. 34123-59-6, Tokyo Chemical Industry, Japan) were treated to 4 weeks cultivated adventitious ginseng roots. The treated samples and their corresponding control were collected at different time intervals $(0 \mathrm{~h}, 1 \mathrm{~h}$, $4 \mathrm{~h}, 8 \mathrm{~h}, 12 \mathrm{~h}, 24 \mathrm{~h}$, and $48 \mathrm{~h}$ ) for qPCR.

\section{RNA isolation and quantitative real-time PCR (qPCR)}

Total RNA was isolated using the RNeasy Plant Mini Kit (Qiagen, Valencia, CA, USA) according to the manufacturer's instructions with modifications. The DNase I (Takara, Japan) treatment was additionally performed before the washing step in a $100 \mu \mathrm{L}$ reaction volume containing $1 \mu \mathrm{L}$ of RNase inhibitor for $1 \mathrm{~h}$. The concentration 
of the extracted RNA was determined using the NanoMD UV-Vis spectrophotometer (Scinco, Seoul, Korea). To synthesize the first-strand of cDNA, total RNA (up to $5 \mu \mathrm{g}$ maximum) was reverse transcribed using the RevertAid reverse transcriptase (Thermo, USA). A qPCR was performed using the Thermal Cycle Dice real-time PCR system (Takara, Shiga, Japan) according to the manufacturer's instructions. The qPCR was performed in a $20 \mu \mathrm{L}$ reaction volume using the following thermal cycling conditions: initial denaturation at $95^{\circ} \mathrm{C}$ for $30 \mathrm{~s}$, followed by 40 cycles of $95^{\circ} \mathrm{C}$ for $5 \mathrm{~s}$ and $60^{\circ} \mathrm{C}$ for $30 \mathrm{~s}$, an additional cycle of $95^{\circ} \mathrm{C}$ for $15 \mathrm{~s}$ and $60^{\circ} \mathrm{C}$ for $30 \mathrm{~s}$, and a final dissociation step at $95{ }^{\circ} \mathrm{C}$ for $15 \mathrm{~s}$. At the end of the qPCR, a dissociation curve was generated to evaluate the generation of by-products. To determine the absolute and/or relative fold-differences in template abundance for each sample, the threshold cycle $(\mathrm{Ct})$ value of each sample was normalized to that of $\beta$-actin, and the formula $2^{-\triangle \Delta \mathrm{Ct}}$ (relative quantification) was used to calculate the gene expression levels. At least three independent experiments were performed in order to validate the $\Delta \Delta \mathrm{Ct}$ method. Dissociation analysis at the end of each run was performed to confirm the specificity of the reaction. The gene-specific primers for $P g C Y P 76 B 93$ were 5'-CAC AAG TCC CTG TCC ACC-3' (forward) and 5'-GTG GTT GTG GAC GTG GAG-3' (reverse). The control primers for ginseng $\beta$-actin (DC03005B05) were $5^{\prime}$-AGA GAT TCC GCT GTC CAG AA-3' (forward) and 5'-ATC AGC GAT ACC AGG GAA CA- $3^{\prime}$ (reverse). Other primer sequences that were used are listed in Additional file 1: Table S1.

\section{Molecular docking study of PgCYP76B93 with isoproturon and chlorotoluron}

The structure of CYP76B93 was predicted by ExPASySWISS MODEL using Zebra Fish CYP-450 17A1 mutant Abiraterone complex (PDB code: 6B82) [21] as major template which exhibited $48 \%$ sequence similarity, because the crystal structure of CYP76B93 have not been defined yet. Molecules that are used to identify interaction with CYP76B93 are isoproturon (CID: 36679) and chlorotoluron (CID: 27375) retrieved from the PubChem database. These molecules were exported as the standard 3-dimentional XML format using the Open Babel 2.4.1. Docking of the two small molecules with CYP76B93 was processed by the AutoDock vina (The Scripps Research Institute, La Jolla, CA, USA) and set as 20 docking poses in the order of the highest negative $\Delta \mathrm{G}$. The flexibility of the ligands was kept as possible and placed at the interface region. The binding possibility of isoproturon and chlorotoluron with CYP76B93 were examined according to their binding energy, and then specific residues involved in interaction with CYP76B93 were determined by LigPlot ${ }^{+}$[22] (The European Bioinformatics Institute).

\section{Vector map construction and in-planta transformation}

The full-length cDNA (1551 bp) of PgCYP76B93 was amplified to functionally characterize in the Arabidopsis heterologous system. The in-planta gene transfer was carried out by using the constitutive $35 \mathrm{~S}$ promoterdriven pCAMBIA1390 [23]. PgCYP76B93 was amplified using the following primers containing added restriction enzyme sites (SalI and MfeI): 5'-AA GTC GAC ATG GAT ATC TTA ACC ATG-3' ${ }^{\prime}$ and $5^{\prime}$-CG CAA TTG AAT CAT AAC TGG AGT TG-3'. The purified PCR product was inserted into pCAMBIA1390 by performing overnight ligation at $16{ }^{\circ} \mathrm{C}$. The $P g C Y P 76 C 9$-ligated construct was confirmed by nucleotide sequencing and transformed into Arabidopsis using Agrobacterium tumefaciens strain C58C1 (pMP90) [24]. The transgenic transformants were selected on hygromycin-containing plates $(50 \mu \mathrm{g} / \mathrm{mL})$, and more than $15 \mathrm{~T} 1$ independent lines were selected. The homozygous transgenic lines carrying one copy of the insert and following a Mendelian segregation ratio were further characterized. For further data analysis, the Col-0 and empty vector lines were used as controls for the PgCYP76B93-overexpressing lines.

\section{Phenylurea herbicide resistance test}

Surface-sterilized seeds were sown on the $1 / 2$ MS media containing two phenylurea herbicide, chlorotoluron. After 2 days of cold stratification at $4{ }^{\circ} \mathrm{C}$, the seedlings were grown at $23^{\circ} \mathrm{C}$ under long-day photoperiod conditions of $16 \mathrm{~h}$ light $/ 8 \mathrm{~h}$ dark. Chlorotoluron was added to the medium at different concentrations for preliminary tests, and a physiological concentration was chosen to show a clear-cut difference in tolerance.

\section{Statistical analysis}

Significance of the statistical analysis was measured based of the determination of Student's t-test using MS excel software.

\section{Results and discussion}

Isolation and identification of ginseng PgCYP76B93 genes All cytochrome P450 (CYP) genes homologous with known CYP family genes were identified from the expressed sequence tag (EST) libraries constructed from the embryogenic calli, 4- and 14-year-old roots, leaves, bud, and methyl jasmonic acid (MeJA)-treated adventitious roots [25]. One EST clone showing similarity with $C Y P 76 B$ family was further chosen, and the recovery of its full-length cDNA was confirmed by rapid amplification of cDNA ends (RACE) polymerase chain reaction (PCR) [26]. The full-length cDNA of $C Y P 76 B$ was 


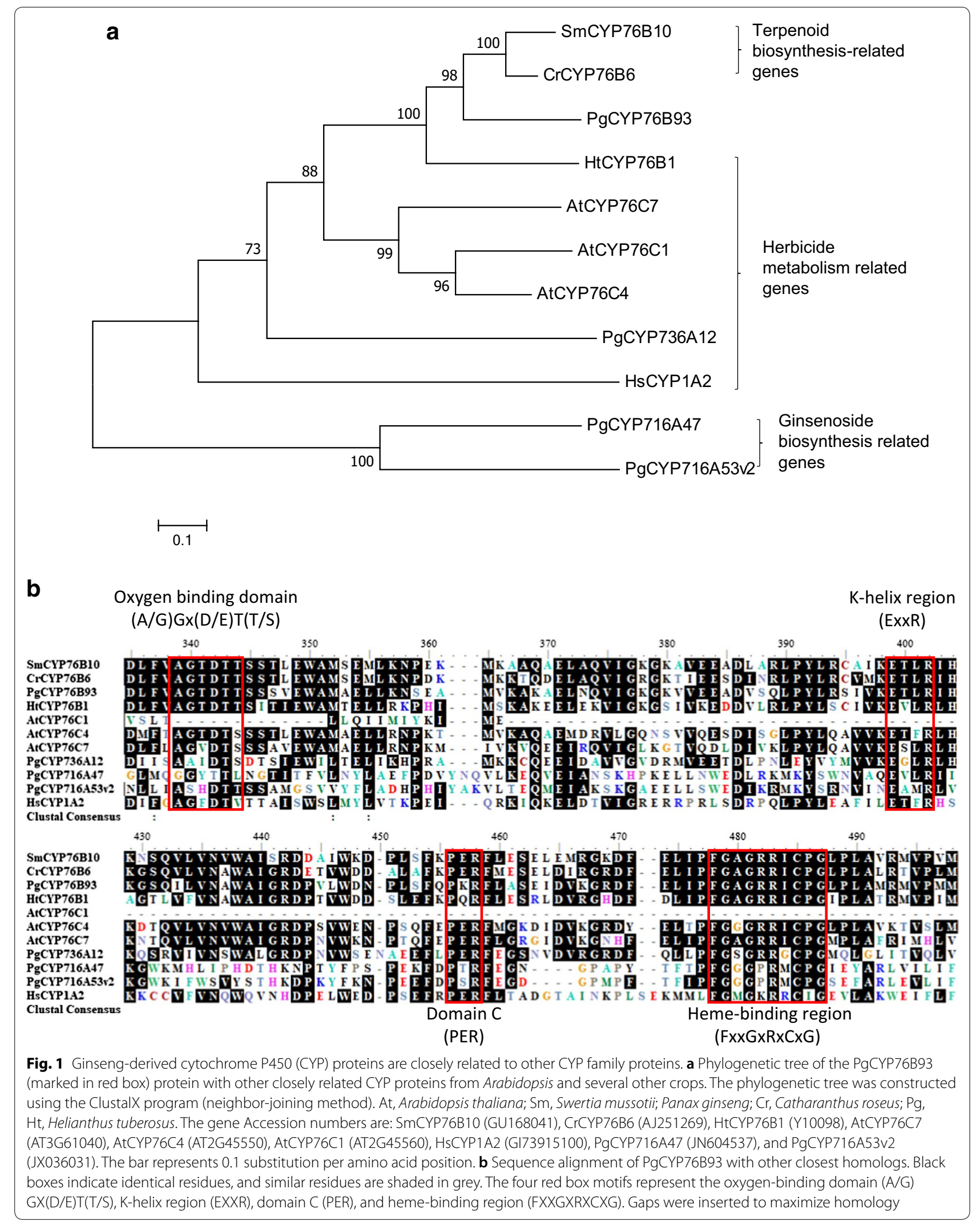


<smiles>Cc1ccc(NC(=O)N(C)C)cc1Cl</smiles>

Chlorotoluron

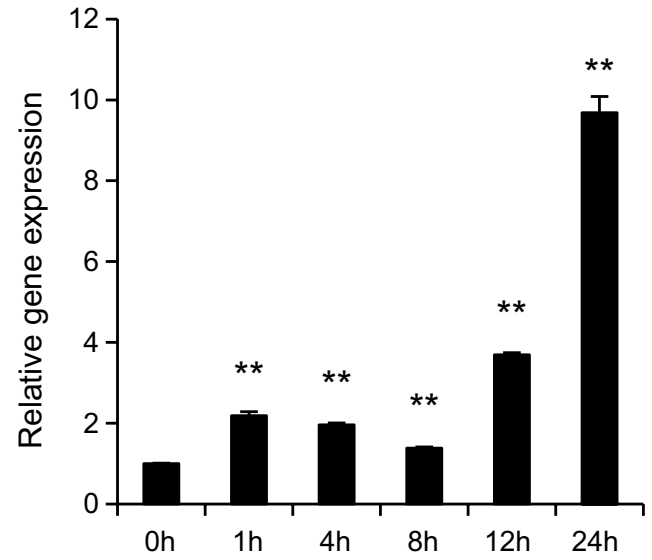<smiles>CC(C)c1ccc(NC(=O)N(C)C)cc1</smiles>

Isoproturon

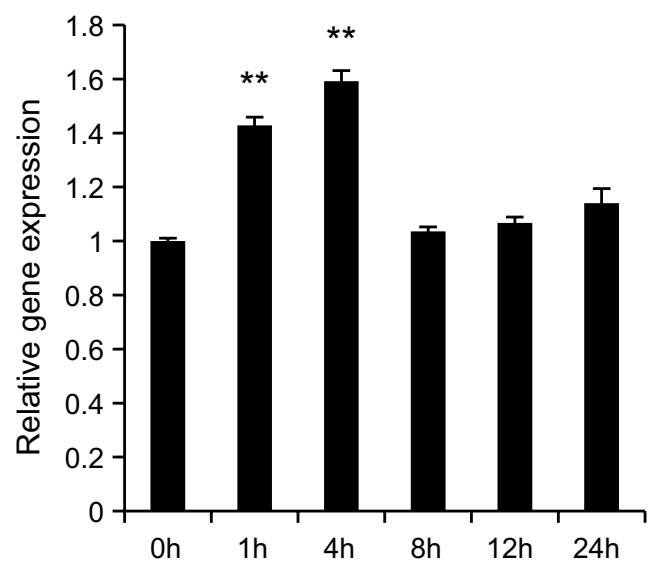

Fig. 2 Temporal gene expression patterns of PgCYP76B93 in response to phenylurea herbicides. Four weeks cultivated ginseng adventitious roots were treated with $10 \mu \mathrm{M}$ of each chlorotoluron and isoproturon for the time intervals indicated and used for the quantification of gene expression. Data represent the mean \pm standard error (SE) for three independent replicates. Means for treated samples were significantly different from the control at $P<0.05\left(^{*}\right)$ and $P<0.01\left(^{* *}\right)$ by Student's t-test, respectively

annotated again as $\operatorname{PgCYP76B93~(CYP76B93~from~}$ $P$. ginseng) after consulting with the CYP nomenclature committee [27]. The original EST clone coding for $P g C Y P 76 B 93$ was obtained from MeJA-treated adventitious roots, indicating that it can be induced by an elicitor and is expressed weakly in normal conditions. $P g C Y P 76 B 93$ is a 1551 bp long gene encoding 517 amino acids. Other amino acid sequences of the characterized CYPs in plants were collected from various gene banks and were used to generate a phylogenic tree (Fig. 1a). PgCYP76B93 was clustered in between the CYP76B family enzymes involved in terpenoid biosynthesis $[28,29]$ and several CYP76B and CYP76C family enzymes [15, $16,18]$, which were previously reported to be involved in phenylurea herbicide resistance (Fig. 1a). The enzymes belonging to the A-type subfamily of the CYP76 family enzymes were clustered relatively closer with PgCYP76B93, and three ginseng CYP enzymes involved in ginsenoside biosynthesis [6-8] were clustered far more distantly (Fig. 1a). This indicates that PgCYP76B93 likely plays a role in herbicide resistance and/or terpenoid biosynthesis.

The PgCYP76B93 enzyme was predicted to have a molecular weight of $58,390 \mathrm{kDa}$ and a $\mathrm{pI}$ of 7.74 using the ProtParam program [30]. The CYP family enzymes were reported to share low similarity in their amino acid sequences, except for several conserved domains, which are necessary for their tertiary structure and enzymatic function [31]. The most well-conserved motif is the heme-binding region FxxGxRxCxG (also known as the CxG motif), in which $C$ (cysteine) binds to the heme group. The ExxR and PER motifs form the E-R-R triad and play important roles in locking the structure of the heme pocket. The most conserved amino acids in the CYP family are glutamic acid (E) and arginine (R) in the ExxR motif and cysteine in the CxG motif, and these amino acids are also found in PgCYP76B93 (Fig. 1b). The least conserved motif, (A/G) Gx(D/E)T(T/S) (also known as AGxDTT), contributes to oxygen binding and activation. The AGxDTT motif is also well conserved in PgCYP76B93, but the PER motif is replaced by the PKR motif (Fig. 1b). Understanding the significance of the substitution of K (lysine) for $\mathrm{E}$ requires further investigation.

\section{Transcript levels of $P g C Y P 76 B 93$ are gradually increased by chlorotoluron}

In one step to understand the function of $P g C Y P 76 B 93$, modulation of transcripts against two phenylurea herbicides were evaluated according to the recent report [20]. Differential mRNA levels of $P g C Y P 76 B 93$ was quantified in time-dependent manner by treatment of $10 \mu \mathrm{M}$ of chlorotoluron and isoproturon (Fig. 2). The transcripts of 

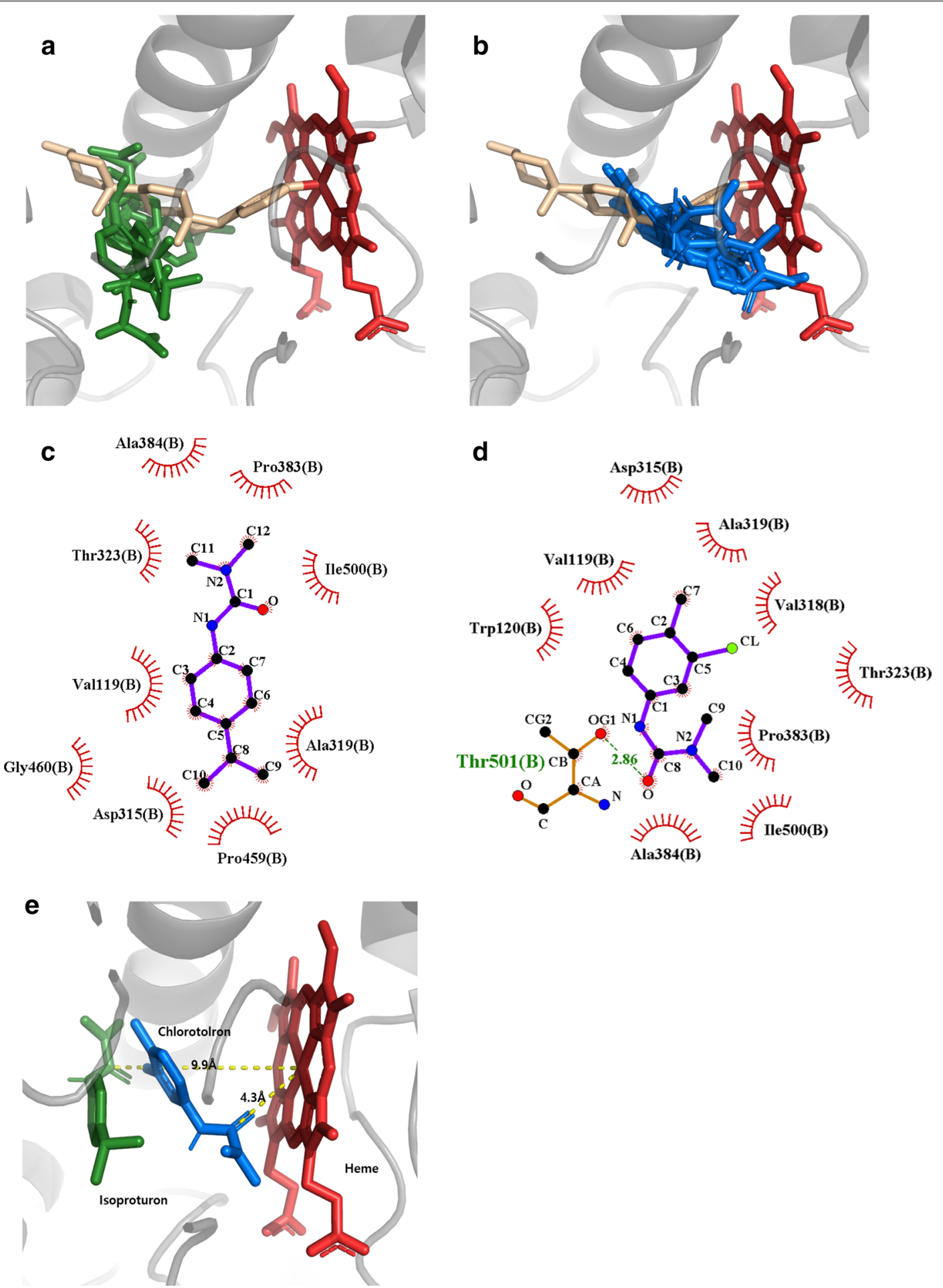

Fig. 3 Isoproturon and chlorotoluron docking near heme site of PgCYP76B93. a Five poses of isoproturon in green, and $\mathbf{b}$ six poses of chlorotoluron in blue are focused from all interactions on predicted PgCYP76B93 overlaying with Zebra Fish CYP-450 17A1 mutant Abiraterone complex. Heme is colored in red stick. Abiraterone is marked in light brown stick. $\mathbf{c}$, d Residues of simulated PgCYP76B93 were expected to interact with isoproturon $\mathbf{c}$ and chlorotoluron $\mathbf{d}$ are presented by using $\mathrm{LigPlot}^{+}$program. Hydrophobic interaction is indicated in spoked arcs labelling with residue, and hydrogen bond is marked in green dotted line with distance $(\AA)$ and residue. Distances from predicted heme of PgCYP76B93 to isoproturon and chlorotoluron are shown in (e). All figures were produced using PyMol (https://pymol.org/2/). 


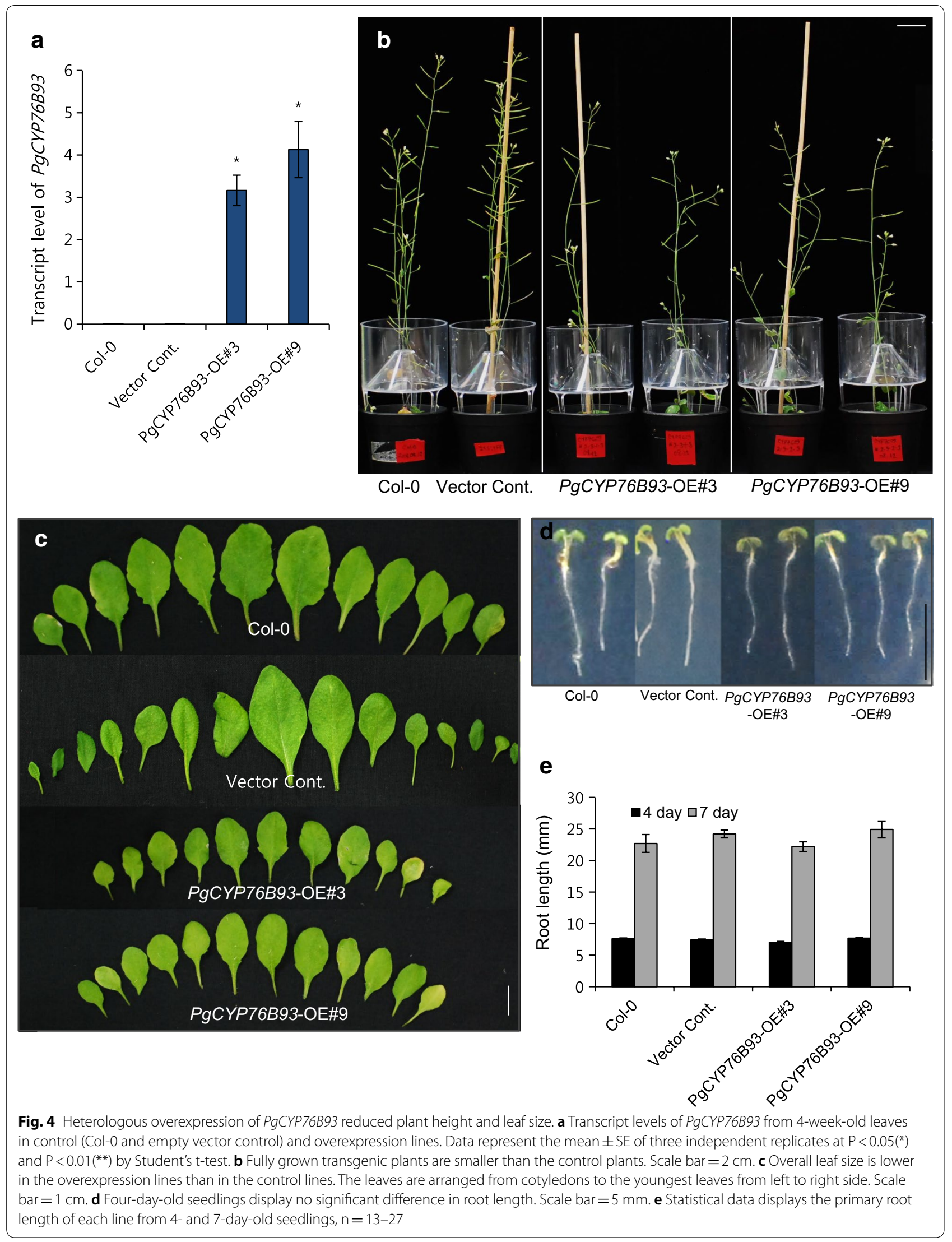


PgCYP76B93 were increased by chlorotoluron gradually from 1 to $24 \mathrm{~h}$ after treatments, and were initially upregulated by isoproturon upto $4 \mathrm{~h}$ (Fig. 2). It indicates that PgCYP76B93 might play roles in herbicide detoxification, especially chlorotoluron.

\section{Molecular docking study shows putative phenylurea herbicide interaction with PgCYP76B93}

The major secondary structure of PgCYP76B93 is pretty identical to Zebra Fish CYP-450 17A1 mutant Abiraterone complex [21]. The heme site of simulated PgCYP76B93 was not appeared but it could be predicted by superimposing the simulated PgCYP76B93 on the Zebra Fish CYP-450 17A1 Abiraterone complex (Additional file 2: Fig. S1). When isoproturon and chlorotoluron were added to the AutoDock program to test the binding possibility with PgCYP76B93, possible docking simulation for the interaction was observed. Total 5 of 20 docking poses for isoproturon and 6 of 20 docking poses for chlorotoluron are resided around heme in active site (Fig. 3a, b). These positions are interestingly located on the similar site of abiraterone which is a substrate of the template protein, Zebra Fish CYP-450 17A1. Residues interacting with isoproturon and chlorotoluron around heme of CYP76B93 was identified by Ligplot+ program [22] (Fig. 3). Isoproturon dominantly forms hydrophobic interaction with Arg102, Val119, Trp120, Met214, Asp315, Val318, Ala319, Thr323, Pro383, Ala384, Leu387, Ile388, Arg456, Pro459, Gly460 and Ile500 in 5 poses, and minor hydrogen bond with Ile388 and Arg390 in 1 poses near heme (Fig. 3c). Chlorotoluron majorly contacts with Val119, Trp120, Asp315, Val318, Ala319, Asp322, Thr323, Pro383, Ala384, Leu387, Ile388, Arg390, Arg456, Ile500 and Thr501 in 6 poses by hydrophobic interaction and minorly interacts with Ile388, Arg390 and Thr501 by hydrogen bond in 3 poses (Fig. 3d). Val119, Trp120, Val318, Ala319, Thr323, Thr384, Leu387, Ile388, Ile500 involved in interaction with isoproturon or chlorotoluron are frequently observed in each poses, so it can be further considered as a target for mutagenesis study. The closest distance from heme to isoproturon is $9.9 \AA$ and to chlorotoluron is $4.3 \AA$ (Fig. 3e). This result is believed to support the data that transgenic Arabidopsis possessing overexpression of CYP76B93 gene exhibits resistance against chlorotoluron other than isoproturon. Closely bound chlorotoluron to heme of PgCYP76B93 might be used as a substrate for undergoing detoxification mechanism.
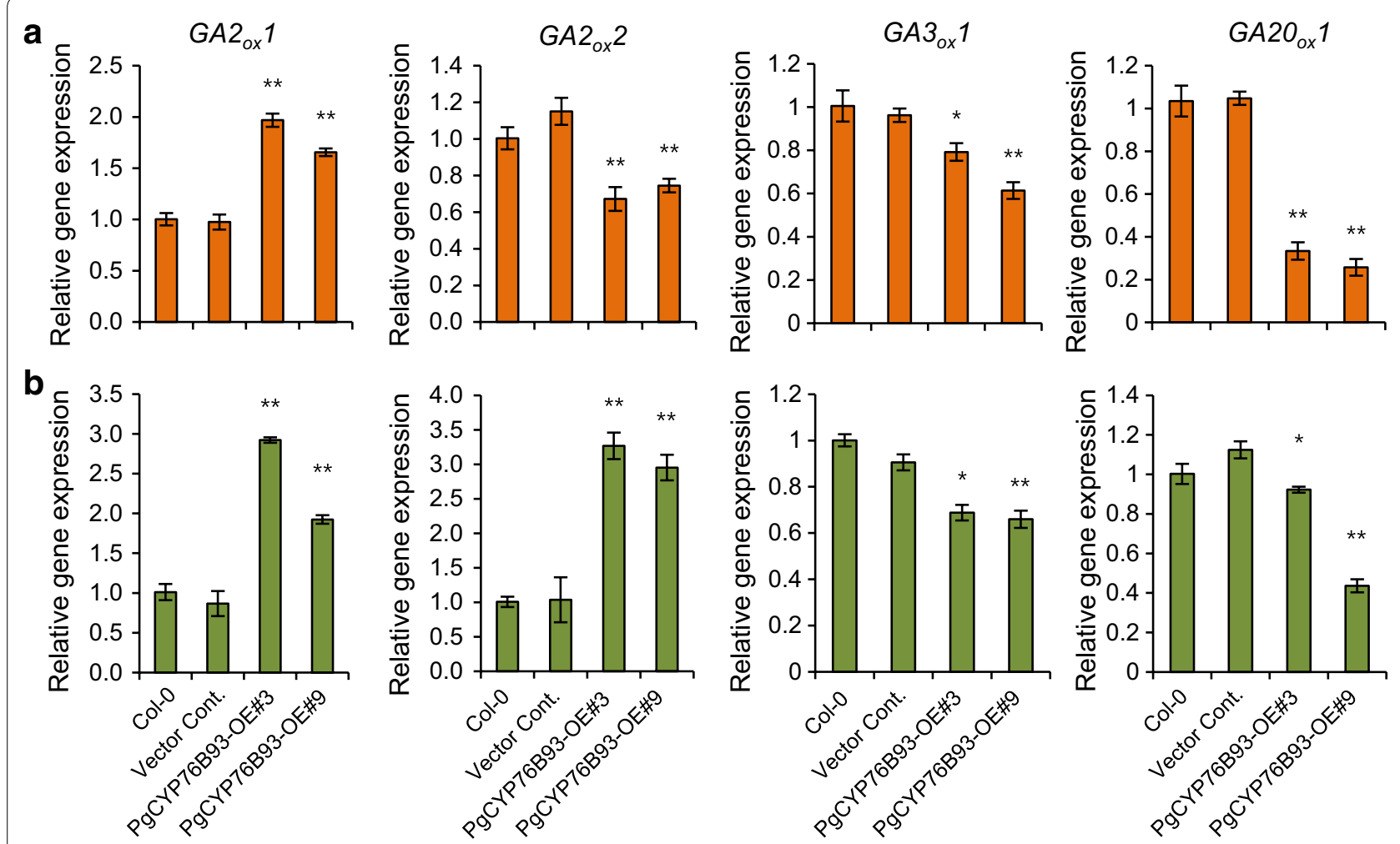

Fig. 5 Gene expression patterns of gibberellin oxidases resulting from the overexpression of PgCYP76B93. Relative gene expression of four gibberellin oxidases (GA20x1, GA20x2, GA30x1, and GA200x1) in 14- a and 28-day-old seedlings b compared to Col-0. Data represent the mean \pm standard error (SE) of three independent replicates at $P<0.05\left(^{*}\right)$ and $P<0.01\left(^{* *}\right)$ by Student's t-test 

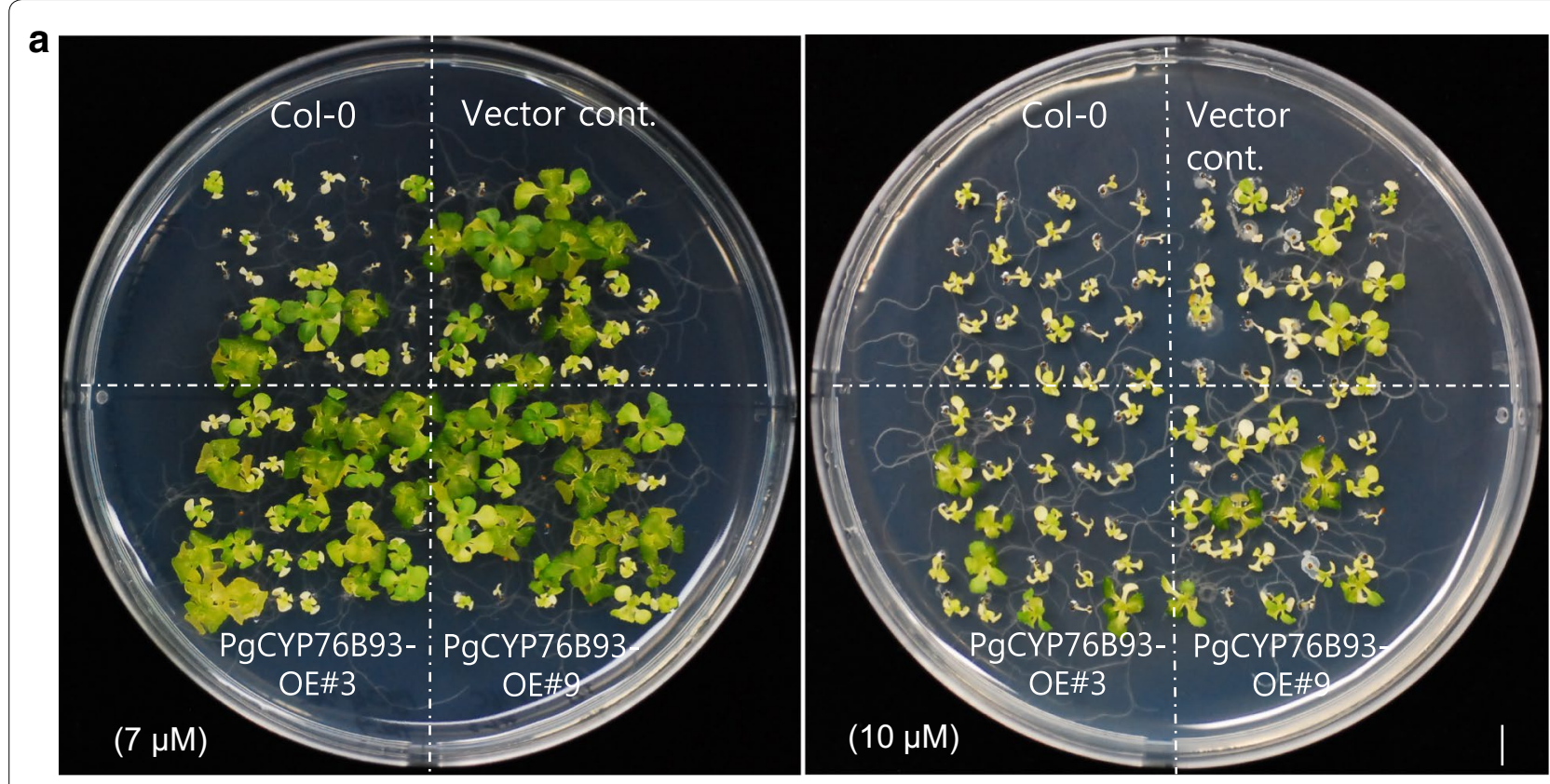

b

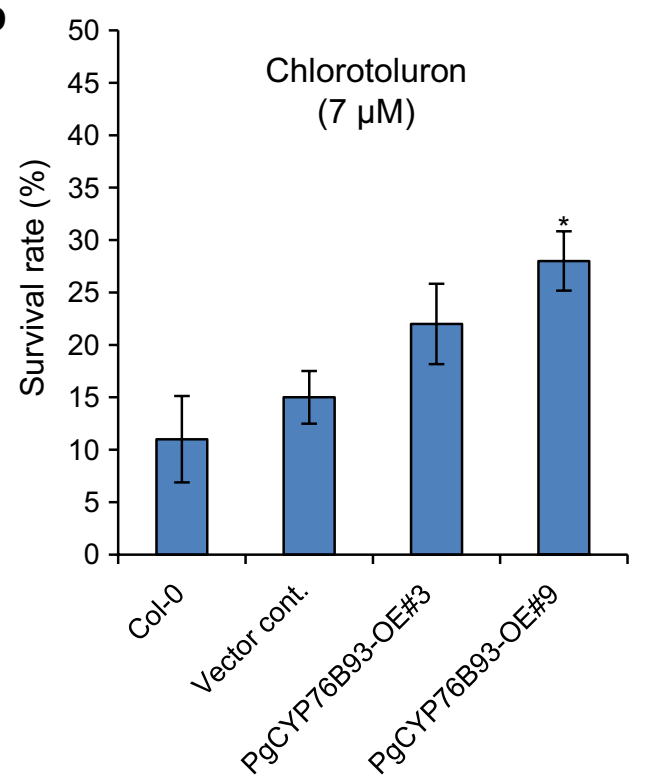

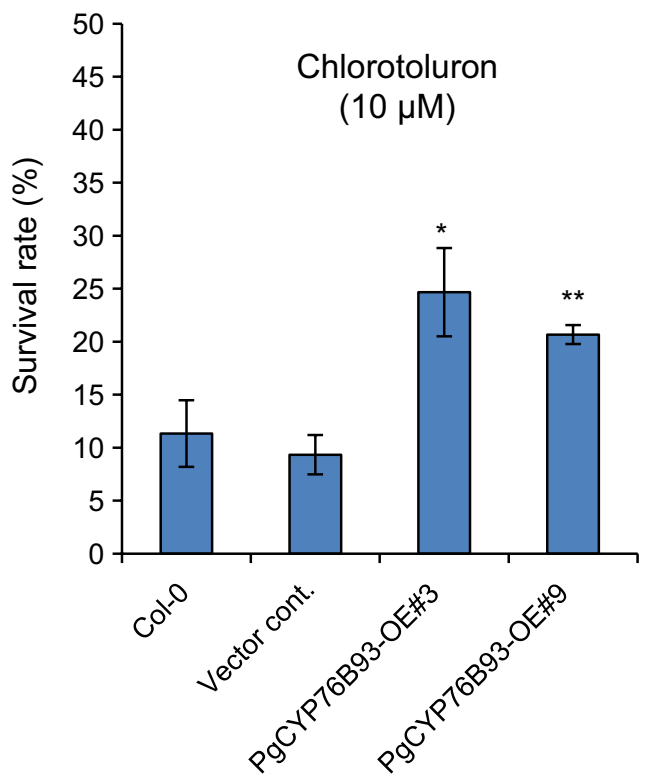

Fig. 6 Overexpression of PgCYP76B93 confers herbicide tolerance. a Arabidopsis Col-0 and transgenic overexpression lines (OE lines) of PgCYP76B93 were germinated on $1 / 2 \mathrm{MS}$ media in the presence or absence of 7 - and $10 \mu \mathrm{M}$ of chlorotoluron for 15 days. $\mathbf{b}$ Statistical data displays the survival rate (\%) of transgenic plants germinated on 1/2 MS media in the presence of 7-and $10 \mu \mathrm{M}$ of chlorotoluron for 15 days. (25 seedlings per plate, total 6 plates were analyzed). Scale bars $=1 \mathrm{~cm}$. Data represent the mean \pm standard error (SE) of three independent replicates at $P<0.05(*)$ and $\left.P<0.011^{* *}\right)$ by Student's t-test

\section{Overexpression of $\mathrm{PgCYP76B93}$ reduced plant height} and leaf size

In order to understand the functional characteristics of PgCYP76B93 in plant growth and development, its fulllength coding sequence was overexpressed in heterologous Arabidopsis plants. Of the several T1 transgenic lines selected on the media containing an antibiotic, two were characterized further. These two transgenic lines showed on average 3.6 times more upregulation of PgCYP76B93 (Fig. 4a) and displayed reduced plant height (Fig. 4b). In seedling stage, one strongly expressing line No.9 displayed reduced hypocotyl length (Additional 
file 2: Fig. S2). It could be resulted in slightly reduced plant height in overexpression lines. Rosette leaves were smaller than those in the control lines (Fig. 4c). This implies that $P g C Y P 76 B 93$ might be involved in plant growth and development via phytohormone regulation which is involved in stem and leaf development. However, the overexpression of $P g C Y P 76 B 93$ did not alter the root growth phenotype (Fig. $4 \mathrm{~d}$, e) suggesting that the phytohormone-regulated $P g C Y P 76 B 93$ gene is more functional in aerial parts than in underground parts.

\section{Transcripts of gibberellin oxidases are altered by the overexpression of $P g C Y P 76 B 93$}

Gibberellins (GAs) are well-known plant hormones that promote stem elongation as well as seed germination [32]. The PgCYP76B93-mediated reduced plant height (Fig. 4b) led us to analyze whether the transcript levels of gibberellin biosynthesis-related genes were changed. Gibberellins are biosynthesized from geranylgeranyl diphosphate (GGDP), which is a common C20 precursor for diterpenoids, using three different classes of enzymes [33]: (1) terpene synthases (2) cytochrome P450 monooxygenases, and (3) 2-oxoglutarate-dependent dioxygenases. To uncover a possible link with bioactive GA biosynthesis, four GA monooxidase genes were analyzed. Two GA oxidase enzymes, GA20ox1 and GA3ox1, are involved in catalyzing the reaction of bioactive GA biosynthesis, whereas GA2ox1 and GA2ox2 are involved in the conversion of bioactive gibberellins into an inactive form. In two different developmental stages, all transcripts of GA20ox1 and GA3ox1 were significantly downregulated, whereas GA2ox1 and GA2ox2 were more upregulated (Fig. 5a, b). Though the mRNA levels of GA2ox2 were higher in the 28-day-old seedlings than in the 14-day-old seedlings, the general tendency was a decrease in the expression of the more bioactive form of GA oxidase gene, suggesting that the reduced height (Fig. 4b) of the PgCYP76B93 overexpression lines is caused by reduction in the levels of the more bioactive forms of gibberellins.

\section{Constitutive overexpression of $P g C Y P 76 B 93$ confers herbicide resistance}

PgCYP76B93 is closely clustered with the CYP76B family of enzymes, which have been reported to be involved in herbicide metabolism [15-17]. Also upregulation of $P g C Y P 76 B 93$ transcripts against chlorotoluron supported this notion. Thus, the PgCYP76B93 overexpression lines were evaluated for their ability to display resistance to phenylurea herbicide chlorotoluron. Several concentrations $(0.5,1,3,7,10 \mu \mathrm{M})$ of chlorotoluron were analyzed from the germination, and the seedlings exposed to 7 and $10 \mu \mathrm{M}$ of chlorotoluron showed distinct tolerant phenotypes compared to the control plants (Fig. 6a). Statistical analysis also suggests that the survival rate (\%) on 1/2 MS media containing herbicide is significant (Fig. 6b). To evaluate the resistance of already germinated seedlings, 4-day-old seedlings from Col-0 and PgCYP76B93 overexpression lines (OE lines) were analyzed in the presence and absence of two different concentrations of chlorotoluron, and found OE lines show apparent tolerant growth phenotype (Additional file 2: Fig. S3).

\section{Supplementary information}

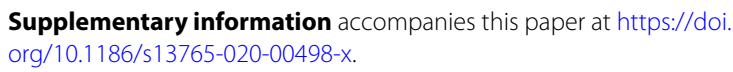

Additional file 1: Table S1. PCR oligonucleotide primers used to confirm gene insertion.

Additional file 2: Fig. S1. Predicted 3D structure of CYP76B93. a The structure of Zebra Fish CYP-450 17A1 mutant Abiraterone complex. It is used as a major template to draw PgCYP76B93. Heme is colored in red, and Abiraterone is colored in light green. $\mathbf{b}$ The theoretical structure of PgCYP76B93. It was derived using ExPASy-SWISS MODEL. This figure was produced using PyMol (https://pymol.org/2/). Fig. S2. Heterologous overexpression of PgCYP76B93 reduced hypocotyl length. Statistical data of hypocotyl length of each lines from 4-day-old seedlings. Data represent the mean \pm standard error (SE) of three independent replicates at $P<$ $\left.0.05{ }^{*}\right)$ by Student's t-test. $n=23$. Fig. S3. Overexpression of PgCYP76B93 confers herbicide tolerance. Four-day-old seedlings of Col-0 and OE lines were transferred on 1/2 MS media in the presence or absence of 4- and 7 $\mu \mathrm{M}$ of clorotoluron for 18 days. Scale bars $=1 \mathrm{~cm}$.

\section{Abbreviations}

CYP: Cytochrome P450 enzyme; qRT-PCR: Quantitative real-time reverse transcription-polymerase chain reaction; $\mathrm{ABA}$ : Abscisic acid; $\mathrm{H}_{2} \mathrm{O}_{2}$ : Hydrogen peroxide; JA: Jasmonic acid; HAT: Hours after treatment.

\section{Authors' contributions}

ORL conceived the project and designed the experiments. JHJ, and SK performed the experiments, except for the molecular docking experiment, which was performed by YKM and SS. ORL and JHJ analyzed the data and wrote the manuscript. All authors read and approved the final manuscript.

\section{Funding}

This study was supported by a Basic Science Research Program through the National Research Foundation of Korea (NRF) funded by the Ministry of Science, ICT, \& Future Planning (Grant number: 2019R1A2C1004140).

\section{Availability of data and materials}

The datasets used and/or analysed during the current study are available from the corresponding author on reasonable request.

\section{Competing interests}

The authors declare that they have no competing interests.

\section{Author details}

${ }^{1}$ Department of Applied Plant Science, College of Agriculture and Life Science, Chonnam National University, Gwangju 61186, Korea. ${ }^{2}$ Interdisciplinary Program of Bioenergy and Biomaterials Graduate School, Department of Bioengineering and Biotechnology, College of Engineering, Chonnam National University, Gwangju 61186, Republic of Korea.

Received: 29 December 2019 Accepted: 18 February 2020 Published online: 29 February 2020 


\section{References}

1. Mizutani M, Ohra D (2010) Diversification of P450 genes during land plant evolution. Annu Rev Plant Biol 61:291-315

2. Kim YJ, Lee OR, Oh J, Jang MG, Yang DC (2014) Functional analysis of 3-hydroxy-3-methylglutaryl coenzyme A reductase encoding genes in triterpene saponin-producing ginseng. Plant Physiol 165:373-387

3. Nguyen NQ, Lee OR (2017) Overexpression of ginseng UGT72AL1 causes organ fusion in the axillary leaf branch of Arabidopsis. J Ginseng Res 41:419-427

4. Nguyen NQ, Lee SC, Yang TJ, Lee OR (2017) cis-Prenyltransferase interacts with a Nogo-B receptor homolog for dolichol biosynthesis in Panax ginseng Meyer. J Ginseng Res 41:403-410

5. Devi BS, Kim YJ, Sathiyamoorthy S, Khorolragchaa A, Gayathri S, Parvin S, Yang DU, Selvi SK, Lee OR, Lee S, Yang DC (2011) Classification and characterization of putative cytochrome P450 genes from Panax ginseng C. A. Meyer. Biochemistry 76:1347-1359

6. Han JY, Kim HJ, Kwon YS, Choi YE (2011) The Cyt P450 enzyme CYP716A47 catalyzes the formation of protopanaxadiol from dammarenediol-II during ginsenoside biosynthesis in Panax ginseng. Plant cell physiol 52:2062-2073

7. Han JY, Hwang HS, Choi SW, Kim HJ, Choi YE (2012) Cytochrome P450 CYP716A53V2 catalyzes the formation of protopanaxatriol from protopanaxadiol during ginsenoside biosynthesis in Panax ginseng. Plant Cell Physiol 53:1535-1545

8. Han JY, Kim MJ, Ban YW, Hwang HS, Choi YE (2013) The involvement of $\beta$-amyrin 28-oxidase (CYP716A52v2) in oleanane-type ginsenoside biosynthesis in Panax ginseng. Plant Cell Physiol 54:2034-2046

9. Werck-Reichhart D, Hehn A, Didierjean L (2000) Cytochrome P450 for engineering herbicide tolerance. Trends Plant Sci 5:116-123

10. Powles SB, Yu Q (2010) Evolution in action: plant resistant to herbicides. Annu Rev Plant Biol 61:317-347

11. McGonigle B, Keeler SJ, Lau SMC, Koeppe MK, O'Keefe DP (2000) A genomics approach to the comprehensive analysis of the glutathione S-transferase gene family in soybean and maize. Plant Physiol 124:1105-1120

12. Brazier M, Cole DJ, Edwards R (2002) O-Glucosyltransferase activities toward phenolic natural products and xenobiotics in wheat and herbicide-resistant and herbicide-susceptible black-grass (Alopecurus myosuroides). Phytochemistry 59:149-156

13. Kebeish R, Azab E, Peterhaensel C, El-Basheer R (2014) Engineering the metabolism of the phenylurea herbicide chlortoluron in genetically modified Arabidopsis thaliana plants expressing the mammalian cytochrome P450 enzyme CYP1A2. Environ Sci Pollut Res Int 21(13):8224-82232

14. Frear DS, Swanson HR, Tanaka FS (1969) N-Demethylation of substituted 3-(phenyl)-1-methylureas-isolation and characterization of a microsomal mixed function oxidase from cotton. Phytochemistry 8:2157-2169

15. Robinau T, Batard Y, Nedelkina S, Cabello-Hurtado F, LeRet M, Sorokine O, Didierjean L, Werck-Reichhart D (1998) The chemically inducible plant cytochrome P450 CYP76B1 actively metabolizes phenylureas and other xenobiotics. Plant Physiol 118(3):1049-1056

16. Didierjean L, Gondet L, Perkins R, Lau SM, Schaller H, O'Keefe DP, WerckReichhart D (2002) Engineering herbicide metabolism in tobacco and Arabidopsis with CYP76B1, a cytochrome P450 enzyme from Jerusalem artichoke. Plant Physiol 130:179-189

17. Höfer R, Boachon B, Renault H, Gavira C, Miesch L, Iglesias J, Ginglinger JF, Allouche L, Miesch M, Grec S, Larbat R (2014) Dual function of the CYP76 family from Arabidopsis thaliana in the metabolism of monoterpenols and phenylurea herbicides. Plant Physiol 166:1149-1161

18. Siminszky B, Corbin FT, Ward ER, Fleischmann TJ, Dewey RE (1999) Expres sion of a soybean cytochrome P450 monooxygenase CDNA in yeast and tobacco enhances the metabolism of phenylurea herbicides. Proc Natl Acad Sci 96:1750-1755

19. Azab E, Kebeish R, Hegazy AK (2018) Expression of the human gene CYP1A2 tolerance and detoxification of the phenylurea herbicide linuron in Arabidopsis thaliana plants and Escherichia coli. Environ Pollut 238:281-290

20. Khanom S, Jang J, Lee OR (2019) Overexpression of ginseng cytochrome P450 CYP736A12 alters plant growth and confers phenylurea herbicide tolerance in Arabidopsis. J Ginseng Res 43:645-653

21. Gonzalez E, Johnson KM, Pallan PS, Phan TT, Zhang W, Lei L, Wawrzak Z, Yoshimoto FK, Eqli M, Guengerich FP (2018) Inherent steroid 17a, 20-lyase activity in defunct cytochrome P450 17A enzymes. J Biol Chem 293(2):541-556

22. Laskowski RA, Swindells MB (2011) LigPlot+: multiple ligand-protein interaction diagrams for drug discovery. J Chem Inf Model 51(10):2778-2786

23. Lee OR, Kim SJ, Kim HJ, Hong JK, Ryu SB, Lee SH, Cho HT (2010) Phospholipase $A 2$ is required for PIN-FORMED protein trafficking to the plasma membrane in the Arabidopsis root. Plant Cell 22:1812-1825

24. Bechtold N, Pelletier G (1998) In planta Agrobacterium-mediated transformation of adult Arabidopsis thaliana plants by vacuum infiltration. Methods Mol Biol 82:259-266

25. Kim MK, Lee BS, In JG, Sun H, Yoon JH, Yang DC (2006) Comparative analysis of expressed sequence tags (ESTs) of ginseng leaf. Plant Cell Rep 25:599-606

26. Pulla RK, Lee OR, In JG, Parvin S, Kim YJ, Shim JS, Sun H, Kim YJ, Senthil K, Yang DC (2011) Identification and characterization of class I chitinase in Panax ginseng C.A. Meyer. Mol Biol Rep 38:95-102

27. Nelson DR (2009) The cytochrome P450 homepage. Hum Genomics 325:68-74

28. Collu G, Unver N, Peltenburg-Looman AM, van der Heijden R, Verpoorte $R$, Memelink J (2001) Geraniol 10-hydroxylase 1, a cytochrome P450 enzyme involved in terpenoid indole alkaloid biosynthesis. FEBS Lett 508:215-220

29. Wang J, Liu Y, Cai Y, Zhang F, Xia G, Xiang F (2010) Cloning and functional analysis of geraniol 10-hydroxylase, a cytochrome P450 from Swertiamussotii Franch. Biosci Biotechnol Biochem 74:1583-1590

30. Wilkins MR, Gasteiger E, Bairoch A, Sanchez JC, Williams KL, Appel RD, Hochstrasser DF (1999) Protein identification and analysis tools in the ExPASy server. Methods Mol Biol 122:531-552

31. Chen W, Lee MK, Jefcoate C, Kim SC, Chen F, Yu JH (2014) Fungal cytochrome p450 monooxygenases: their distribution, structure, functions, family expansion, and evolutionary origin. Genome Biol Evol 6:1620-1634

32. Lester DR, Ross JJ, Davies PJ, Reid JB (1997) Mendel's stem length gene (Le) encodes a gibberellin $3 \beta$-hydroxylase. Plant Cell 9:1435-1443

33. Yamaguchi S (2008) Gibberellin metabolism and its regulation. Annu Rev Plant Biol 59:225-251

\section{Publisher's Note}

Springer Nature remains neutral with regard to jurisdictional claims in published maps and institutional affiliations. 